\title{
Mental retardation and perception of global motion
}

\author{
ROBERT FOX and STEPHEN OROSS III \\ Vanderbilt University, Nashville, Tennessee
}

\begin{abstract}
We have found that mildly mentally retarded adults are impaired in their perception of global stereoscopic forms (Fox \& Oross, 1988) in ways that cannot be attributed to peripheral visual deficits or failures to comprehend. To assess the generality of that result, we measured the ability of mentally retarded adults to perceive kinematographic forms. Mentally retarded and nonretarded adults were presented with a two-choice, forced-choice detection task requiring the location of a target's spatial position. The discriminability of the forms was varied by systematic reductions in both element density and temporal correlation. We found that, relative to nonretarded adults, mentally retarded adults exhibited large qualitative deficits in their ability to discriminate these kinematographic forms when either density or correlation was reduced. After considering a number of alternative interpretations of these data based on factors such as peripheral visual impairment and a failure to attend, we could find none more compelling than a perceptual interpretation, which posits a deficit within the short-range motion system.
\end{abstract}

The purpose of this paper is to report the results of our investigation of the ability of mildly mentally retarded adults to detect a form produced by the coherent motion of a subset of elements in a random-element kinematogram. Persons with this level of retardation perform within the 50-70 IQ range on standard tests of intelligence. These individuals can care for their personal needs and perform simple industrial tasks, under supervision. Usually, the etiology of the intellectual impairment is not known and organic or neurological deficits are assumed not to be present. Our interest in the present investigation stems directly from our prior work, which examined the ability of retarded persons to perceive veridically stereoscopic forms produced by random-element stereograms (Fox \& Oross, 1988). Because many readers of this journal may not be familiar with that study, some background information is in order.

First, our interest in the perceptual abilities of retarded persons was sparked, in part, by our association with the John F. Kennedy Center, ${ }^{1}$ a multifaceted institute at Vanderbilt University that is dedicated to research on mental

This research was supported, in part, by Grants EY00590 from the National Eye Institute and HD07226 from the National Institute of Child Health and Human Development. We would like to express our deep appreciation to Bennett Bertenthal and Steven Kramer for making the computer program available to us and for their help in implementing our testing procedure. We also thank Al Baumeister and other colleagues at the John F. Kennedy Center for their generous slipport and encouragement throughout the project. We are grateful for the specific comments made on an earlier version of this manuscript by a number of our colleagues, including Randy Blake, Mike Braunstein, Richard Held, Joseph Lappin, Ennio Mingolla, and Ken Nakayama and three anonymous referees. We thank the staff and clients of New Horizons, Inc. and Prospect, Inc. for their generous assistance. Reprint requests should be addressed to Robert Fox, Department of Psychology, 301 Psychology Building, Vanderbilt University, Nashville, TN 37240. retardation and human development. Furthermore, our interest in research on global stereopsis and global motion made us aware of the theoretical analysis of these phenomena emanating from the field of artificial intelligence and computational vision. These efforts (e.g., Grimson, 1981; Hildreth, 1984; Marr, 1982; Ullman, 1979) have revealed that the formation of stable and veridical percepts from minute randomly arrayed elements is a complex process that imposes substantial demands on the computational resources available to neural systems. These demands, however, are inferred from theoretical considerations and are not apparent from phenomenal observation. Observers with normal vision uniformly report that they perceive global stereoscopic and motion percepts quickly and effortlessly without the participation of attention or other cognitive processes.

Intrigued by the juxtaposition of theoretical complexity and phenomenal simplicity, we developed the following conjecture. If the random element stimuli do impose substantial computational burdens, then retarded persons, who are often regarded as possessing limited informationprocessing or computation resources, may have difficulty perceiving them effectively.

We tested that conjecture in the three experiments that are described in Fox and Oross (1988). Summarizing the results of that study, we found that retarded persons could identify the left-right spatial location of a stereoscopic form well above chance when the density of the dichoptic random elements was equal and at full or maximum values (e.g., 50\% density for each of the matrices). But as density was reduced, performance declined substantially. This is in marked contrast to the performance of nonretarded persons, who can perceive forms clearly even when density is reduced to values on the order of $1 \%$. They report that deleted elements are replaced by a 
phenomenal filling-in that produces subjective contours that maintain the integrity of the form. We also found that retarded subjects encountered difficulty in discriminating correctly stereoscopic forms of varying shape. Moreover, these subjects did not exhibit depth constancy-the depth position of the stereoscopic form did not follow the position predicted by the geometry of stereopsis (for an analysis, see Cormack \& Fox, 1985a, 1985b). Together, these results suggest that although the retarded persons could discriminate the left-right spatial location of a stereoscopic form, they perceived it as a blob with poorly defined contours that appeared to be localized at a fixed depth position slightly in front of the display.

These results are quite surprising in that substantial deficits in basic perceptual processes in mildly mentally retarded persons have not been reported previously. Moreover, nonretarded persons, including those used as control subjects in the Fox and Oross (1988) study, perceive such stimuli automatically and without error. The pattern of results exhibited by the retarded subjects, however, can be related to the computational model of global stereopsis developed by Marr and Poggio (1979), which consists of three serially ordered stages (primal sketch, 21/2-D sketch, and 3-D model), each of which carries out increasingly refined analyses.

In terms of the model, the ability of the subjects to detect the position of the form indicates that the correspondence problem was resolved at the primal sketch stage. The impairment produced by density reduction and the errors in shape discrimination, however, implies a deficiency at the level of the $21 / 2-D$ sketch, while the failure to localize depth position suggests a corresponding failure at the level of the full 3-D model.

It should be emphasized, however, that the parallel between these data and the stages of the Marr-Poggio model do not imply any special commitment to it. Other computational approaches to vision are available to which our results are relevant. These include the comprehensive theoretical program pursued by Grossberg and his colleagues (e.g., Grossberg, 1987a, 1987b; Grossberg \& Marshall, 1989; Grossberg \& Mingolla, 1987), in which it is assumed that information is multiplexed among interdependent processes rather than processed by modular stages such as the $21 / 2$-D sketch.

But our inquiry has not been motivated primarily by theoretical considerations. Indeed, we introduced the concept of computational vision simply to provide some theoretical framework that might encompass the two seemingly diverse domains of interest embraced by this investigation. One is intelligence, and the many cognitive processes attendant to it. The second is perception, particularly those early stages that are independent of cognitive influence. At the behavioral and phenomenal level, the separation of these domains seems both reasonable and natural. Moreover, it has been argued that the distinction between cognition and perception is a fundamental one
(Fodor, 1983). Yet, at the level of mechanism, neural networks engaged in computation can be used to generate or represent both perceptual and cognitive functions. It would be surprising, given all evidence from evolutionary biology, if common properties were not present at the network level.

But further theoretical development awaits more complete empirical specification of the ability of mentally retarded persons to perceive veridically various kinds of stimuli over a range of relevant conditions. To that end, we examined perception of global motion produced by kinematograms, because of the significant similarities between them and random element stereograms-similarities well documented in the contemporary literature on motion perception (e.g., Anstis, 1978; Nakayama, 1985).

One of the more fundamental similarities is that both kinds of stimuli require resolution of the so-called correspondence problem-elements within the arrays of random elements must be appropriately matched or identified, across either time (kinematograms) or space (stereograms). When the correspondence condition is satisfied, forms are perceived that appear to have distinct edges and palpable surfaces. Yet, these percepts depend completely on the successful processing of more fundamental stimulus dimensions, either motion or stereopsis. Moreover, these kinetic and stereoscopic forms are similar in terms of their resolution limits defined by spatial and temporal transfer functions (e.g., Nakayama \& Tyler, 1981; Rogers \& Graham, 1982). It is of special interest that the perceptibility of kinetic forms, like their stereoscopic counterparts, is not impaired by substantial reductions in element density (Baker \& Braddick, 1982; Gilden, Bertenthal, \& Othman, in press; Regen \& Hong, 1990).

To assess perception of a kinetic form generated by a kinematogram, we used a simple task in which the subject indicated the spatial position, left or right, of the form. Two stimulus variables-elemental density and temporal correlation, based on the percentage of elements that define the form and are correlated over time-were varied jointly.

\section{METHOD}

\section{Subjects}

The subjects were 9 mildly mentally retarded ( 5 females, 4 males) and 6 nonretarded ( 2 females, 4 males) young adults. The mentally retarded subjects were paid volunteers $(\$ 5 / \mathrm{h})$, recruited from a population of day workers enrolled in sheltered workshop programs. Their mean age was 33.7 years (range $=25.7-47.9$ ). Their mean IQ was 58.3 (range $=50-70$ ). With the exception of 1 Down Syndrome subject, the etiology of their retardation was not known. An additional 8 mentally retarded subjects were excluded from the study because they were unable either to respond to the randomelement kinematograms, despite their being able to accurately respond to a practice form $(n=5)$, or to comprehend the task instructions $(n=3)$. The nonretarded subjects were graduate students, staff members, or visitors to the laboratory. Their mean age 
was 26.5 years $($ range $=23.1-31.7$ ). Their mean IQ was unknown but was clearly in the normal range. Three of these subjects were inexperienced as psychophysical observers and were naive as to the purpose of the study; the other 3 subjects were experienced as psychophysical observers.

Both groups of subjects possessed normal or corrected-to-normal vision, as determined by either a modified version of a test of contrast sensitivity (Vistech) or the illiterate E test. Furthermore, all of the subjects were free of obvious binocular impairments, such as gross misalignment of the eyes.

\section{Apparatus and Stimuli}

The apparatus used to generate and display the random-element kinematograms consisted of an AT\&T 6300 Plus microcomputer and an AT\&T RGB monitor with a 12 -in. raster. The resolution of the display shown on the monitor was 640 pixels (width) $\times 200$ pixels (height). Individual frames of the kinematograms were precalculated and stored in memory, thus enabling their sequential presentation during the experiment.

The stimuli were two-frame random-element kinematograms in which each frame was presented for $17 \mathrm{msec}$ and followed by an interstimulus interval of $34 \mathrm{msec}$. The test stimulus consisted of a rectangular form whose elements were vertically displaced as a correlated unit across frames. It was superimposed on a background whose elements were uncorrelated across frames and possessed the same average displacement as the displacement of the correlated elements. Thus, the test stimulus appeared as a rectangle vertically oscillating over a background of random motion that resembled video noise or Brownian motion. Two variables were manipulated, element density and correlation. Element density varied in octaves (approximately) and could assume values of $50 \%$ (half of the potential elements were filled), $25 \%, 12 \%, 6 \%, 3 \%$, or $1 \%$. (At $50 \%$ density, 1,200 elements were contained in the test stimulus: At $25 \%$ density, 600 elements were contained in the test stimulus, and so forth.) Correlation ranged from $100 \%$ (all of the elements in the rectangular form were displaced the same amount in the same direction) to $10 \%$ in $10 \%$ steps. The background and the test stimulus were always presented with the same element densities. Figure 1 presents a schematic representation of the test stimulus and provides the specifications of the physical properties.
In addition, as a control condition, a practice stimulus was employed that possessed the same specifications as the test stimuli except that it appeared as a black rectangle superimposed on a randomly moving background that had a density of $50 \%$.

\section{Procedure}

The subjects were seated $160 \mathrm{~cm}$ from the monitor and introduced to a two-alternative spatial forced-choice task in which, under free viewing conditions, they were required to indicate the left/right spatial position of the kinematographic form. To determine if subjects could comprehend the task, they were required to discriminate the position of the practice stimulus over 16 trials. All did so without error.

The overall psychophysical method followed the dictates of the descending method of limits wherein the difficulty of stimulus discrimination proceeds from easy to hard. One modification, however, consisted of reintroducing an easier to discriminate stimulus after the subject had encountered a difficult discrimination. This was done for two reasons: (1) It served to maintain motivation, and (2) it served to ensure that the difficulty in discrimination was due to the specific stimulus condition under test and not attributable to fatigue or other nonperceptual factors.

In the formal execution of the experiment, the subjects were presented with a stimulus of a given element density selected on a random basis. This stimulus, with a correlation of $100 \%$, was presented for 16 trials. If the stimulus was discriminable on at least 12 trials, the correlation was reduced by $10 \%$ and the next set of 16 trials was administered. When the number of correct responses fell to less than 12 , the experimenter applied the reintroduction procedure described earlier.

\section{RESULTS}

In Figure 2, the results (coded as percent correct) obtained from both the mentally retarded and nonretarded groups are illustrated as a function of temporal correlation and element density. In Figure 3, the results obtained for individual subjects for those same variables are illus-

\section{Displacement $=8.59$ arc $\mathrm{min}$}

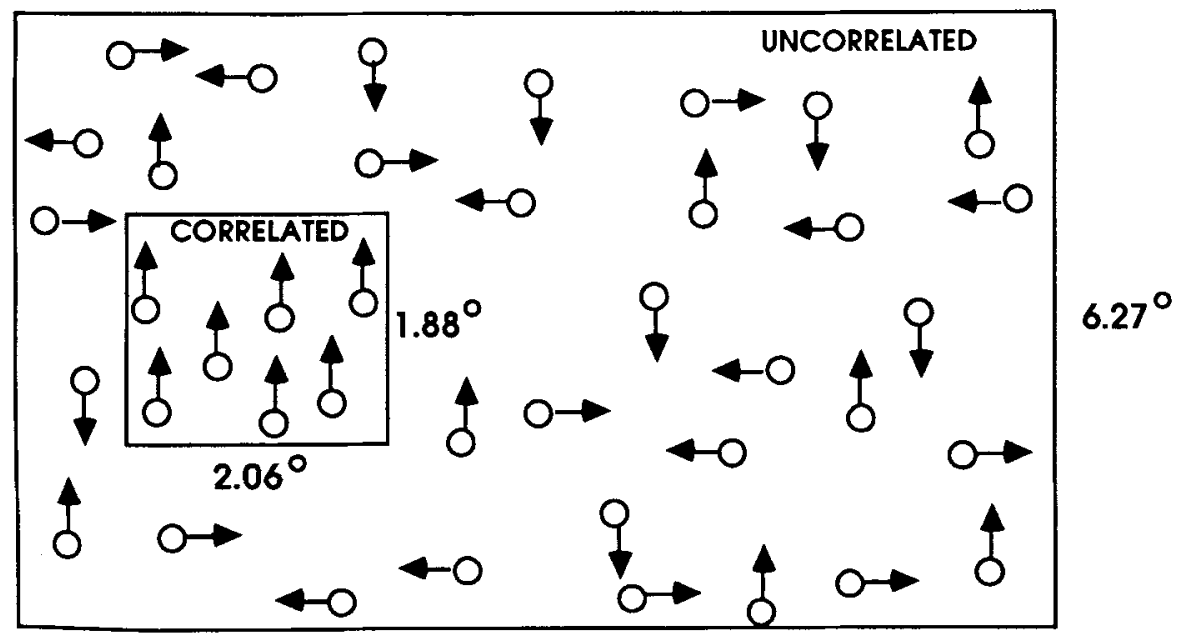

$8.24^{\circ}$

Figure 1. Schematic representation of the random-element kinematogram. Across trials, the correlated region could appear either on the left half (as depicted) or on the right half of the monitor. 

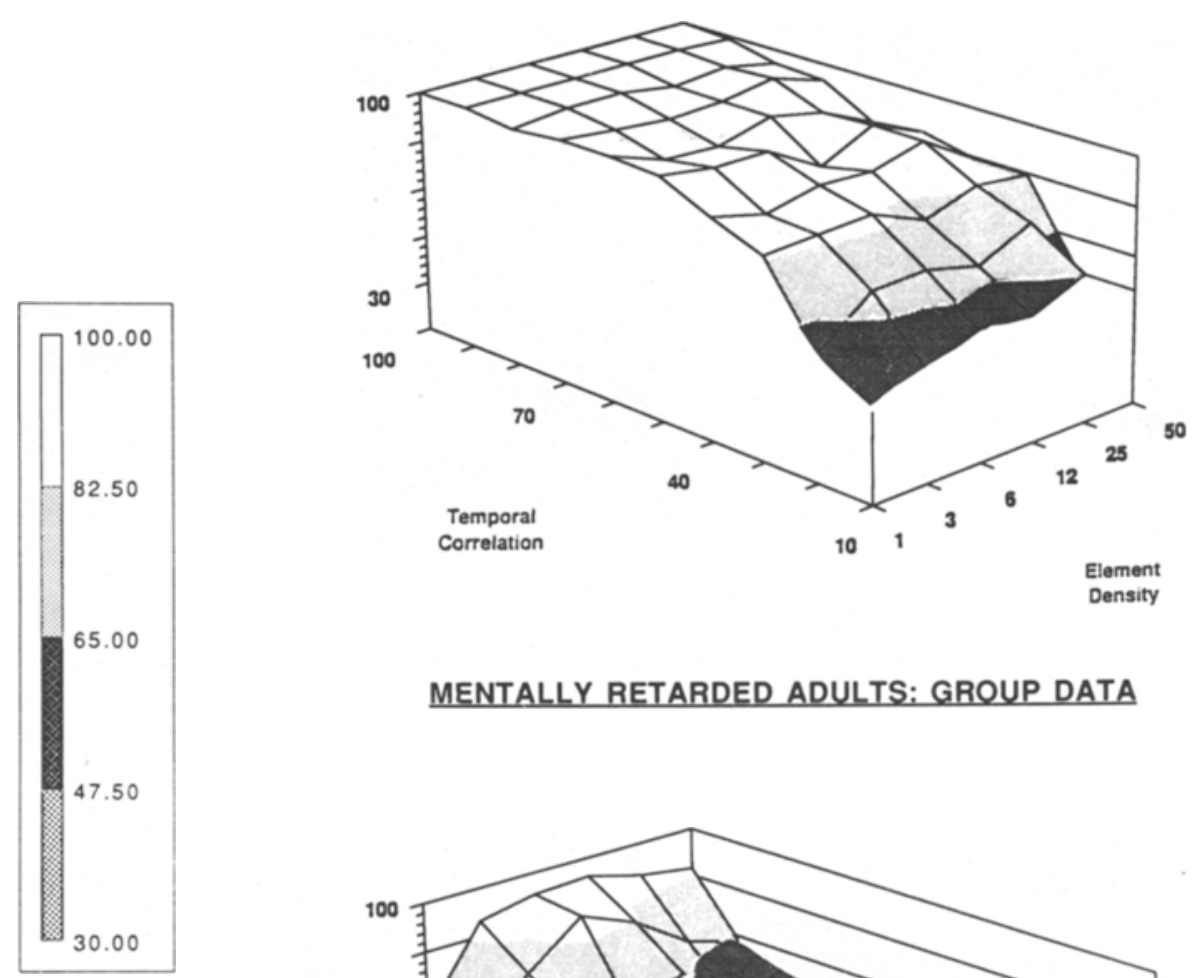

\section{MENTALLY RETARDED ADULTS: GROUP DATA}

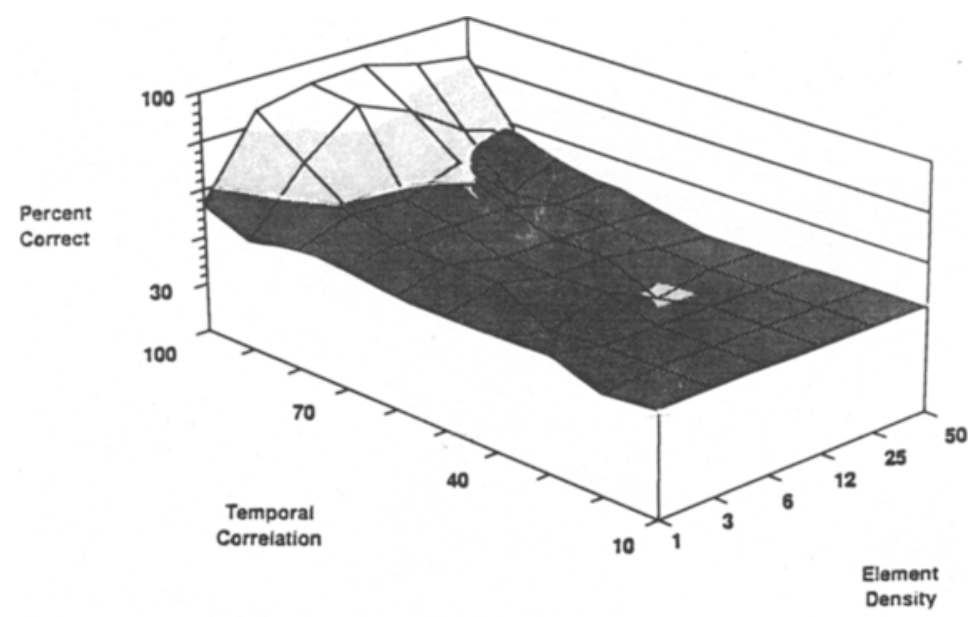

Figure 2. Three-dimensional representation of the group data obtained from nonretarded and mentally retarded adults. The percentage of correct responses is displayed as a function of both element density and temporal correlation (Note: $50 \%$ correct is chance performance.)

trated. As can be clearly seen, there are large differences in performance between the two groups, with the performance of the mentally retarded group rapidly declining to chance at temporal correlation levels of at least $60 \%$. For that reason, our statistical analyses were confined to correlation levels of $60 \%$ and higher.

Separate 6 (density) $\times 5$ (correlation level) ANOVAs were conducted for the mentally retarded and nonretarded groups because of the near ceiling levels of accuracy dis- played by the nonretarded group, which resulted in limited variance. Additionally, the Greenhouse and Geisser epsilon adjustment in degrees of freedom was applied for all analyses to minimize Type I errors. The results of the analyses for the nonretarded group revealed no significant effects for density $[F(5,25)=1.82, p>.05]$, correlation $[F(4,20)=2.94, p>.05]$, or their interaction $[F(20,100)=1.22, p>.05]$. The results of the analysis for the mentally retarded group, however, did reveal 
NONRETARDED ADULTS: INDIVIDUAL SUBJECT DATA
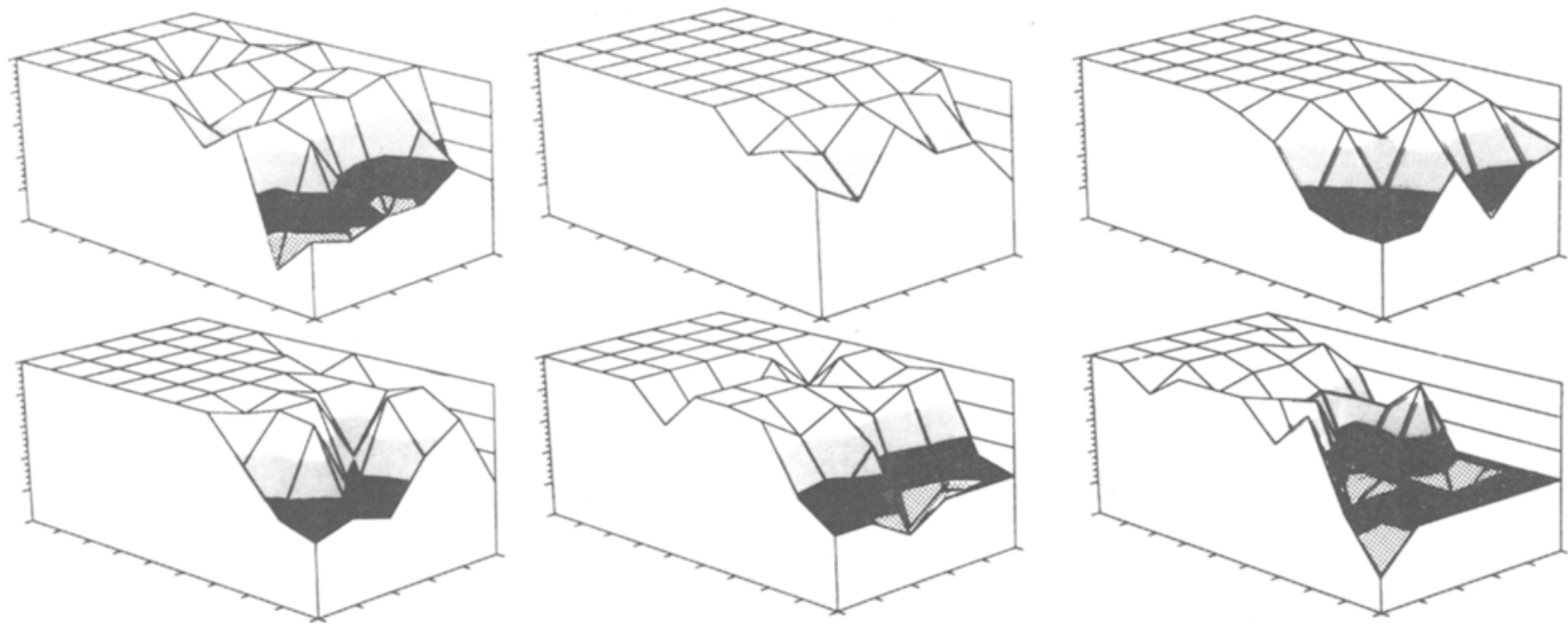

MENTALLY RETARDED ADULTS: INDIYIDUAL SUBJECT DATA
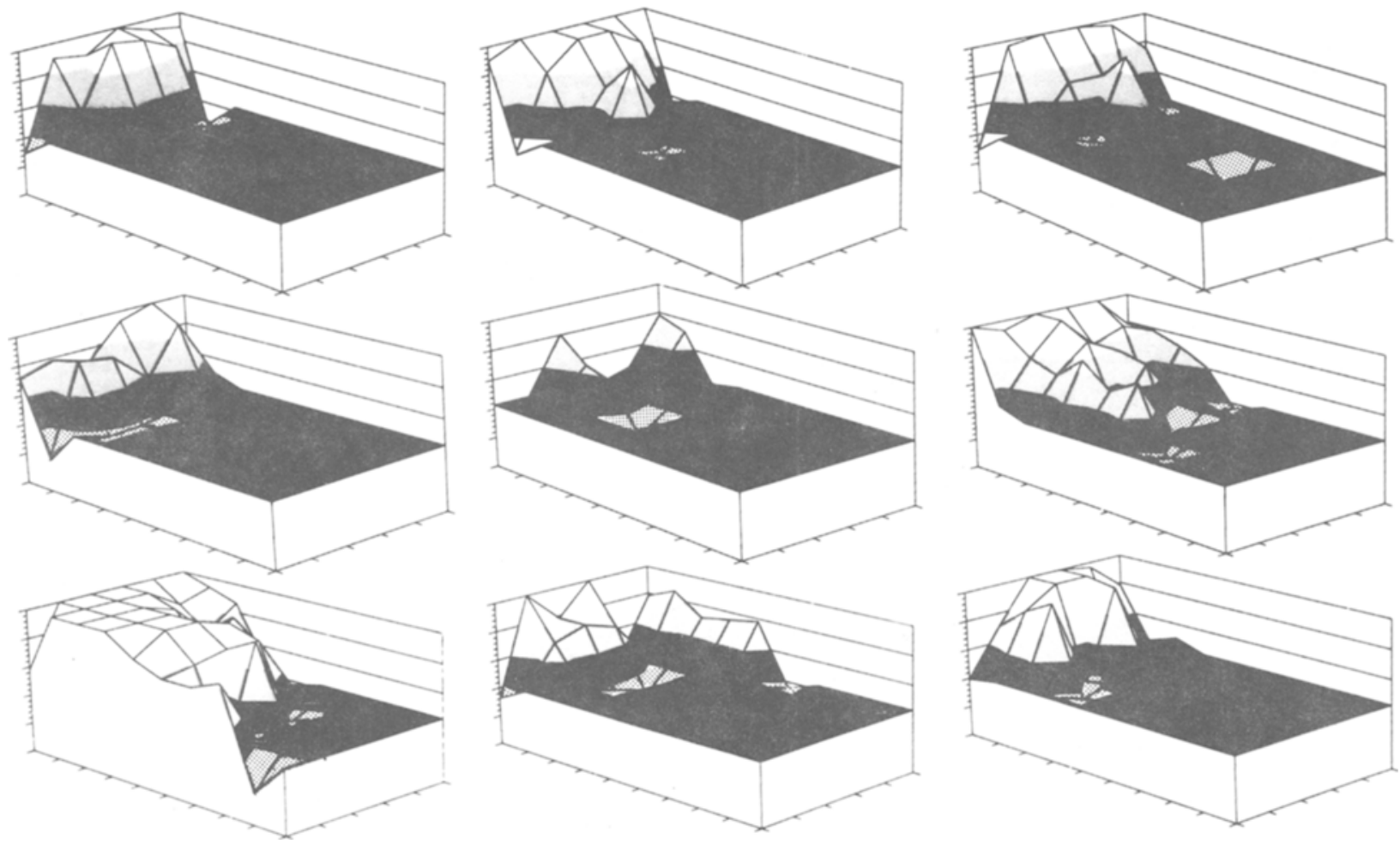

Figure 3. Three-dimensional representation of the individual data obtained from nonretarded and mentally retarded adults. The axes are identical to those shown in Figure 2. 
significant effects for density $[F(5,40)=6.71$, $p<.002]$, correlation $[F(4,32)=31.85, p<.0001]$, and their interaction $[F(20,160)=2.76, p<.03]$.

\section{DISCUSSION}

The pattern of results depicted in the three-dimensional graphs given in Figures 2 and 3 indicates that, relative to the performance of nonretarded subjects, the performance of retarded subjects was profoundly impaired by reductions in density and correlation. This relationship is supported by the statistical analysis of the group performance described in the Results section. Although performance of individual subjects was not analyzed statistically, the graphic presentation of individual performance given in Figure 3 makes it clear that the differences between groups are not based on the results of a few aberrant subjects. Rather, there appears to be a qualitative difference between the retarded and nonretarded subjects. This is made clear by the results of the analysis that is confined to performance at correlations of $60 \%$ and higher. Within that range, the performance of nonretarded subjects is essentially perfect, as demonstrated by the absence of significant main effects for either density or correlation. Over the same range of those variables, however, the performance of retarded subjects declines substantially, as demonstrated by the significant main effects for both density and correlation. Indeed, retarded subjects performed at chance under stimulus conditions that elicited error-free performance from the nonretarded subjects.

Although the impaired performance of the retarded subjects is quite substantial, the interpretation of its basis warrants careful analysis. The application of standard criteria would place the deficit unequivocally within the domain of perception. It is well established that the stimuli are processed at an early stage within the visual system (for a review, see Petersik, 1989). The psychophysical method and experimental design are ones known to minimize the contribution of nonperceptual response factors. In brief, all the criteria that define a proper experiment in perception have been fulfilled.

Nevertheless, before interpreting the deficit solely in perceptual terms, the feasibility of alternative interpretations will be considered. This caution is prompted by the impaired intellectual status of the retarded subjects, which could have acted in some way to impede performance.

Failure to comprehend the task is one alternative interpretation that should be considered. But evidence against it comes from the successful performance of the subjects under conditions of high stimulus density and correlation. Furthermore, it is significant that subjects could perform successfully in easier stimulus conditions, after encountering more difficult conditions. Taken together, the results suggest that the behavior of the subjects was controlled by the conditions of stimulation, and this in turn requires comprehension of the task.

Could anomalies of the peripheral visual system, such as low acuity, be responsible for the deficits? This is a question often posed. Several considerations support a negative answer. First, the estimates of acuity and/or contrast sensitivity that we obtained from the subjects in this study fell within normal limits. Even if these clinical tests are imperfect, they would be capable of detecting gross impairments in vision. It should be noted, parenthetically, that as a group, the visual capacities of mildly retarded persons do not differ from the distribution of such capacities found in a general nonretarded population (O'Dell, Harshaw, Abernathy, Pool, \& Boothe, 1988). But even if the visual acuity of the subjects had been impaired, it is unlikely that this would have impaired detection performance. Direct evidence on this point comes from a study by Oross, Carlin, and Fox (1990b) on the effect of dioptic blur on kinetic form detection under stimulus conditions very similar to those used in this study. They found that even high levels of blur (approaching 4 to 6 diopters) did not significantly impair the performance of their nonretarded subjects. These results are consistent with those in which functional blur is introduced by spatial frequency filtering that leaves only elements with the lowest frequencies remaining in a display (e.g., Gilden et al., in press). Finally, the question of binocular anomalies of vision does not arise because the motion phenomena can be readily seen with a single eye-indeed, one with rather poor focus. Taken together, these considerations argue strongly against an alternative interpretation couched in terms of peripheral visual anomaly.

A somewhat more complex alternative interpretation that should be considered is one based on the concept of inappropriate deployment of attention or some similar kind of cognitive strategy. On this hypothesis, the high performance of the retarded subjects under stimulus conditions of high density and correlation occurred because the stimuli were quite salient and placed no demands upon attention. But as stimuli became more difficult to discriminate because of reductions in density and correlation, more attention, in some sense, was required for detection. Presumably, the retarded subjects were less able to attend to the relevant cues than were the nonretarded subjects. According to this hypothesis, the performance of the retarded subjects should improve markedly after they have had the opportunity to learn to attend to the relevant cues. To gain some insight into the viability of hypotheses of this general type, Oross, Carlin, and Fox (1990a) investigated the extent to which the subjects in this study could benefit from training.

Two kinds of training procedures were used. In one, called fading, the test stimulus was initially made quite discriminable by introducing large differences in density between it and the background; then, over training trials $(n=96)$, discriminability was gradually reduced. In the second procedure, called repeated exposure, the stimulus was unchanged and presented over trials $(n=96)$. For each procedure, the subject was required, on each trial, to indicate the left-right position of the stimulus. These procedures were applied to two kinds of stimulus conditions, one that initially yielded approximately $75 \%$ correct detections and one that initially yielded approxi- 
mately $50 \%$ correct detections (chance responding). These conditions were selected or adjusted for each subject to match their particular performance level. For the stimuli that initially yielded $50 \%$ correct detections, no improvement was found in a posttest that followed the training sessions for either training procedure. For stimuli initially discriminable at $75 \%$ correct detections, some relatively small improvement in performance (approximately $10 \%$ ) was observed. This improvement, however, did not manifest itself suddenly. Rather, it was incremental across training sessions and was restricted to the repeatedexposure training procedure.

The small and gradual improvement found in this study does not seem to offer much support for any particular version of the attention-deployment hypotheses. Although predictions may vary according to the specific set of assumptions used to formulate such a hypothesis, all would seem to require a notable enhancement of performance after administration of training. Instead, the changes in performance that were observed are similar to those routinely seen in threshold experiments in perception and are often attributed to perceptual learning.

Although other alternative interpretations may exist that we have not considered, it is difficult to make a convincing case for any that we have examined. The absence of credible alternatives supports an interpretation of the results in terms of a deficit within the perceptual system. Support for that interpretation can also be derived from the results of the Fox and Oross (1988) study, which used different subjects and quite different procedures, and found analogous effects of density reduction in randomelement stereograms.

If the perception interpretation is correct, then, in the case of motion, the deficit would reside within that part of the motion system known as the short-range process. A fundamental characteristic of this process, which has been subjected to considerable experimental examination (see Petersik, 1989, for a review), is that it integrates the motions of individual elements, linking them together with a kind of perceptual glue that maintains the phenomenal integrity of the kinetic form despite large reductions in density. The integration proceeds automatically, however, quite independent of attention and cognitive processes.

The presence of considerable information about the short-range process should facilitate specification of the presumptive perceptual deficit we have observed. For instance, if the deficit involves the integrative process, the ability of mildly retarded subjects to identify the shapes of kinetic forms should be greatly impaired even when the forms remain detectable. The evaluation of this hypothesis and others, all yoked by the logic of converging operations, should make it possible to evaluate unequivocally the validity of the perceptual interpretation of the deficit.

\section{REFERENCES}

ANSTIS, S. M. (1978). Apparent movement. In R. Held, G. W Leibowitz, \& H.-L. Teuber (Eds.), Handbook of sensory physiology: VIII. Perception (pp. 655-673). Berlin: Springer-Verlag.
BAKER, C. L., \& Braddick, O. J. (1982). The basis of area and dot number effects in random dot motion perception. Vision Research, 22, 1253-1260.

Cormack, R., Fox, R. (1985a). The computation of disparity and depth in stereograms. Perception \& Psychophysics, 38, 375-380.

CoRmack, R., \& Fox, R. (1985b). The computation of retinal disparity. Perception \& Psychophysics, 37, 176-178.

Fobor, J. A. (1983). The modularity of mind. Cambridge, MA: MIT Press.

Fox, R., \& Oross, S. (1988). Deficits in stereoscopic depth discrimination by mildly mentally retarded adults. American Journal of Mental Retardation, 93, 232-244.

Gilden, D. L., Bertenthal, B. I., \& Othman, S. (in press). Image statistics and the perception of apparent motion. Journal of Experimental Psychology: Human Perception \& Performance.

Grimson, W. E. L. (1981). From images to surfaces. Cambridge, MA: MIT Press.

GrossberG, S. (1987a). Cortical dynamics of three-dimensional form, color, and brightness perception: I. Monocular theory. Perception \& Psychophysics, 41, 87-116.

Grossberg, S. (1987b). Cortical dynamics of three-dimensional form, color, and brightness perception: II. Binocular theory. Perception \& Psychophysics, 41, 117-158.

GrossberG, S., \& Marshall, J. (1989). Stereo boundary fusion by cortical complex cells: A system of maps, filters, and feedback networks for multiplexing distributed data. Neural Networks, 2, 29-51.

Grossberg, S., \& Mingolla, E. (1987). Neural dynamics of surface perception: Boundary webs, illuminants, and shape-from-shading Computer Vision, Graphics, \& Image Processing, 37, 116-165.

HildRETH, E. C. (1984). The measurement of visual motion. Cambridge, MA: MIT Press

MarR, D. (1982). Vision. San Francisco: W. H. Freeman.

MARR, D., \& Poggio, T. (1979). A computational theory of human stereo vision. Proceedings of the Royal Society of London, Series B, 204, 301-328.

NaKaYAMA, K. (1985). Biological image motion processing: A review. Vision Research, 25, 625-660.

Nakayama, K., \& Tyler, C. W. (1981). Psychophysical isolation of movement sensitivity by removal of familiar position cues. Vision Research, 21, 427-433.

O'Dell, C. D., Harshaw, K. B., Abernathy, C., Pool, L. G., \& Boothe, R. G. (1988). The assessment of vision in a mentally retarded population. Investigative Ophthalmology \& Visual Science Supplement, 29, 431 .

Oross, S., III, Carlin, M. T., Fox, R. (1990a). Motion perception deficits in mildly mentally retarded adults: An experimental attempt to ameliorate deficits via training. Gatlinburg Conference on Research \& Theory in Mental Retardation \& Developmental Disabilities Abstracts, 23, 66.

Oross, S., II, Carlin, M. T., * Fox, R. (1990b). Short-range apparent motion: Investigations of detection and figural integrity. Investigative Ophthalmology \& Visual Science Supplement, 31, 519.

Petersix, J. T. (1989). The two-process distinction in apparent motion. Psychological Bulletin, 106, 107-127.

REGEN, D., HoNG, X. H. (1990). Visual acuity for optotypes made visible by relative motion. Optometry \& Visual Science, 67, 49-55.

ROGERS, B., \& GraHAM, M. (1982). Similarities between motion parallax and stereopsis in human depth perception. Vision Research, 22, 261-270.

Ullman, S. (1979). The interpretation of visual motion. Cambridge, MA: MIT Press.

\section{NOTE}

1. R.F. holds an appointment as a Kennedy Center Senior Scientist and the time this study was conducted S.O. held an appointment as a Postdoctoral Fellow in the Kennedy Center training program on research in mental retardation.

(Manuscript received August 3, 1989; revision accepted for publication April 8, 1990.) 